\title{
Solar Powered Dc Nano-Grid with Multi Agent Control Strategy
}

\author{
Periyasamy Muthuvel
}

\begin{abstract}
Smart homes are typical examples of DC Nano-grids wherein multi-agent strategy is required for coordinating different entities to harness flexible load and storage to maximize the integration of intermittent renewable generation. This paper proposes a novel multi-agent approach for DC Nano-grids in smart homes with an aim to simultaneously maximize comfort levels and renewable integration. In the proposed approach, there are three agents: flexible loads, batteries, and renewable energy sources which interact among them for meeting the control objectives. The agents are coordinated using a centralized controller and based on its decision the flexibility is harnessed to the grid. The novelty of the approach is that the different agents communicate only to the central controller and not among themselves which reduces the communication among them. The advantage of the proposed approach is their ability to handle $D C$ Nano-grids and using an agent-based approach within a residential building. The proposed multi-agent approach is illustrated on a lab-level DC Nano-grids pilot developed by the authors. Our results show that achieves maximum overall energy efficiency and minimum electricity bill and smooth control of various modes of operation.
\end{abstract}

Keywords: DC Nano-Grid, Multi agent system, smart Home appliances.

\section{INTRODUCTION}

$\mathrm{E}$ xisting power is hastily changing. Presently, the power depends on fossil fuel, coal, and nuclear power generation. But these power grids are nowadays facing a various number of difficulties. Which, integrally, they don't have the necessary action to resolve this issue [1]. And, the usage of long-distance transmission line power distribution causes to lead the lines losses and it reduces the existing grid's efficiency [2]. The power generators like fossil fuel and coal are frequently emitting the carbon dioxide to the atmosphere [3]. Another important problem is estimated at around 1.2 billion people worldwide do not have access to electricity from the existing grid [4]. Because most of them live in isolated places, to where extending the grid is uneconomical [5]. And, the use of alternative energy sources like renewable energy (solar, wind) to satisfy the increasing global load.

But these are intermittent resources. Therefore, incorporating these intermittent resources with the existing grid requires a new coordination controlling methods. Which leads to the controller complexity, and require additional power electronics components. The depletion of fossil fuel and coal resources and increasing the use of renewable energies has motivated to develop a new concept. A new concept is known as "Nano-grids" aims to alter this. This alleviates the need for long distance transmission, improving the grid efficiency and forming the robust system. Because the Distributed Generation (DG) producing power close to the Nano-grids [6]. The above-mentioned problems are overcome by the Nano-grids system. In this Nano-grids, Energy Management System technique is one of the major challenges. This is mainly because households and commercial buildings are representing a major part of electricity consumption [7] and partly because a small range of distributed power sources such as Photo-Voltaic (PV), Battery storages, Wind turbines, and fuel cell have been installed in houses and office. It is introducing substantial complexity in the operation of a Nano-grids. Even though, it can yield distinct benefits to the total system performance, if managed and synchronized efficiently. Significant research is currently carried out regarding operation and control of Nano-grids [8], [9].

In this paper, a multi agent control strategy is proposed, aimed at the following: i) Efficient use of local distributed renewable resources ii) Feeding of household loads based on priority iii) Make operation simplicity. There are various agent levels of decentralization that can be possibly applied, ranging from centralized control to a fully distributed control strategy. According to the fully distributed agent control strategy implemented in this paper, the primary responsibility is given to the agent controllers, which compete to maximize the generation in order to satisfy the load demand, possibly provide the maximum self-consumption. Furthermore, the multi agent strategy should take all the appropriate decisions to ensure safe and smooth energy management operation of the distributed energy resources they are controlling. The organization of a controlled, smart entity formed by several fewer smart entities can be based on multi agent strategy. The multi agent strategy is the evolution of the traditional distributed technology with some particular characteristics that provide new abilities in controlling complex systems [10]. This strategy has already developed in the operation of large power systems such as microgrids [11], [12], [13], [14], [15]. The use of multi agent control strategy in a Nano-grids provide effective results for a number of particular operational problems. For example, household loads, solar power generation, grid connection and storage units have different agents and several decisions should be taken locally.

Revised Manuscript Received on December 15, 2019.

* Correspondence Author

Periyasamy Muthuvel*, Department of Instrumentation and Control Engineering, Kalasalingam Academy of Research and Education, Virudhunagar, Tamilnadu, India. Email: muthuvel.p@klu.ac.in. 
The paper presents a multi agent control approach for Nano-grids. These systems operate inside the varies agent systems and, for that overall atmosphere presented first. Five agent levels are distinguished: - Source agent - Battery storage agent $\bullet$ Grid agent $\bullet$ Load agent $\bullet$ Nano-grids Central controller agent. The source agent is responsible to get maximum power from the $\mathrm{PV}$ at various atmosphere conditions. Second, Battery agent is used to connect or disconnect the battery to the DC link depending upon state of charge of battery, charging or discharging condition of the battery. Third, Grid agent is used to connect or disconnect the main grid to the Nano-grids depending upon the available power and load demand. Fourth, load agent is used connect or disconnect the loads to the Nano-grids and also its scheduling load based on the priority of the loads and available power. Finally, the central controller agent should take all the decisions to maximize the self-consumption and reduce the power import from the grid connection. The overview of the paper is as follows: In section II the description of the Nano-grids is presented. In section III the multi agent control strategy is introduced shortly with some of its benefits for the control of Nano-grids. Section IV, provides the modes of operation with multi agent control strategy for the proposed system, as well as given some pseudo code of various agents in section V. section VI, provides the simulation results description of its multi agent control strategy implementations and section VII conclusion.

\section{PROPOSED DC NANO-GRID SYSTEM CONFIGURATION}

The Proposed DC Nano-grid configuration is shown in Fig.1. It consists of solar PV panel as the main power source, battery to store power during excess solar power availability and to discharge during insufficient power to meet the load demand, dc link connected to three phase ac grid through bidirectional converter and transformer. Battery is directly connected to the dc link having 24 volts nominal voltage. Solar PV panel is connected to dc link through dc/dc buck converter. Pulses are given to the buck converter by source agent which always tracks maximum power point of PV panel. Battery is connected or disconnected to the DC link by battery agent. Similarly, three phase ac grid is also turned on or off by grid agent. 24-volt dc loads are connected directly to the dc link, while 12-volt dc loads are connected through 24/12-volt dc-dc buck converter.

\section{A. Energy Storage}

Energy storage is crucial for balancing supply and demand. As we use solar PV cells, the entire DC Nano-grid system should operate in such a way that the PV panel will maneuver as the source of DC Nano-grid during the day, while the energy stored in the energy storage device during the daytime will operate as the source of DC Nano-grid during the night. Battery will function as a good energy storage device for such a scheme. Because it has the smallest energy density, it can supply elevated power density and therefore its power to-weight ratio is high, lead acid battery is used in the suggested scheme. It is also cheap, low self-discharge and low maintenance requirements compared to other standard batteries.

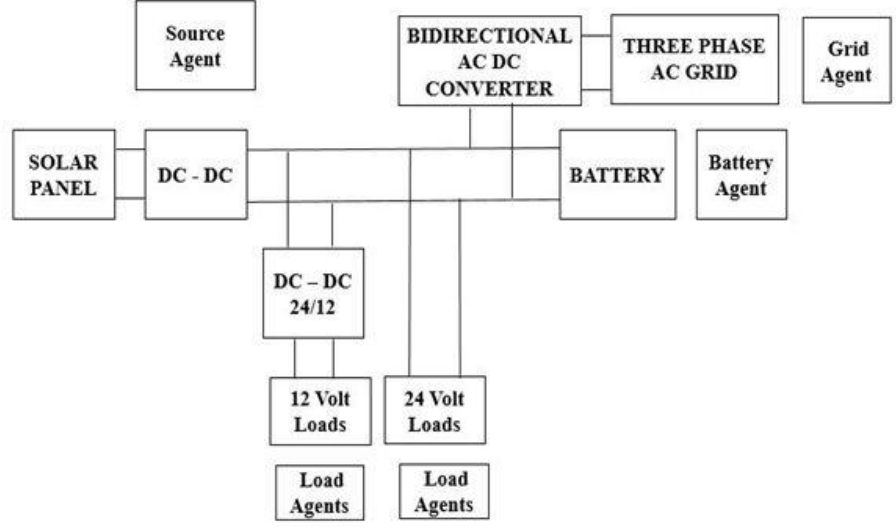

Fig. 1. DC Nano grid proposed system.

\section{B. Bi-directional converter}

Bidirectional power converter regulates the power flow between dc nano grid and utility grid. Voltage Source Converter is used as a bi-directional converter to connect three phase ac grid to the dc link. If Vag leads Va converter operates in rectifier mode i.e. three phase ac grid is feeding power into the dc link. If Va leads Vag converter operates in inverter mode i.e. DC link is feeding power into the three-phase ac grid.

- controller of Bi-directional Converter: Hysteresis current mode controller is a two-loop controller. The outer loop includes a PI controller which maintains continuous DC voltage and the inner loop includes three hysteresis controllers for the sinusoidal shaping of the current and the maintenance of the unit power factor at the three phase ac grid terminals. Filter inductance reduces current harmonics. Instantaneous dc voltage is compared with the reference dc voltage which generates error signal. This error signal is given to PI controller which generates control signal. Control signal is multiplied by three phase voltages in three separate multipliers which generates three reference current signals. These reference current signals are compared with instantaneous three-line currents and generated control signal is given to hysteresis controller. Hysteresis is a ON/OFF controller having a dead band. The output of the hysteresis controller is either fully $\mathrm{ON}$ or fully OFF and these on off pulses are provided to specific gate drivers. If a row current drops below a fixed level in the converter, the corresponding IGBT is switched on and the device turns off the corresponding IGBT once it increases above the set level. Thus, the inner loop makes the input current of the converter sinusoidal and in step with the voltage, whereas the outer loop makes the dc voltage constant at the specified value.

\section{DC NANO GRID CONTROL}

To enhance the performance and operation of these Nano grids, a proper control and communication strategy has to be adopted. There are two main kinds of control techniques to incorporate the renewable power source, three stage ac grid and power storage into the nano grid. The first is centralized control, implemented in. 
The advantage of centralized control is that energy management policies can be readily implemented in the main controller since this controller is conscious of each source and load element throughout the system. In order to generate operational instructions and commands, centralized control needs real-time feedback from each terminal. The significant drawback of such a control system is that the quick switched converters need a high- speed communication route. In view of the quick and non- coincidental demand for load in the housing implementation, even high-speed communication could not meet. If we want to connect a new load, to control automatically need to change this load in the centralized controller. Another kind of control is decentralized control, or independent control. One of those frequently used strategies is the Multi Agent System (MAS) model. Among the multiple control strategies available, the most effective and frequently used is the MAS model. A perfect agent is outlined as a reactive, proactive and flexible software and/or hardware. Connection of a new load with its own load agent is easy in multi agent system rather than centralized system.

\section{MULTI AGENT STRATEGY FOR DC NANO-GRID}

B As for the explanation of a causal agent, the same holds the explanation of a MAS. Different definitions have been suggested based on the discipline from which it originates. Once again, the goal here is not to itemize and refine different definitions, but instead of to choose a one definition that seems to be generalized and close to the research group of power engineering. A multi-agent system is described as, "A MAS is a loosely coupled network of problem-solving entities (agents) that work together to find answers to problems that are beyond the individual capabilities or knowledge of each entity (agent)". A MAS is the set of agents working together to pursue specific duties. The primary characteristic of a MAS is its capacity to monitor and interact in the field with control facilities. The concept behind any MAS scheme is to break down a complicated issue handled by a single entity - a centralized system - into smaller, easier issues handled by several organizations - a distributed system. The fact that the agents within a MAS scheme work together means the involvement of some type of collaboration between individual agents. However, the notion of collaboration in MAS is at best uncertain and at worst highly inconsistent, so that the terminology, doable classifications, etc. are even more difficult than in the situation of agents, which makes any effort to present MAS a difficult issue. A hierarchy of collaboration seems to be the easiest, and here we begin with this hierarchy as the basis for MAS classification. The topology is shown in Fig. 2.

A MAS is autonomous if each singular agent pursues its own goals independently of the other. A MAS is discreet if it is autonomous, and if the agents' objectives have no relationship to each other. Discrete MAS does not require collaboration.

However, officials can collaborate without any intention to do so and if this is the case then the collaboration is emerging. The term thoughtful in figure 2 is not to be assorted with the term of the profound agent, and in the figure 2 it shows to the

idea that agents plan their actions jointly in order to get together with each other. Cooperation within a MAS can be achieved in three respects:

- By explicit design (the agent developer deliberately designs the behaviors of the agent so that collaboration takes place).

- By adapting (individual agents learn to collaborate).

- By evolution (the collaboration of individual agents evolves through some kind of evolutionary process).

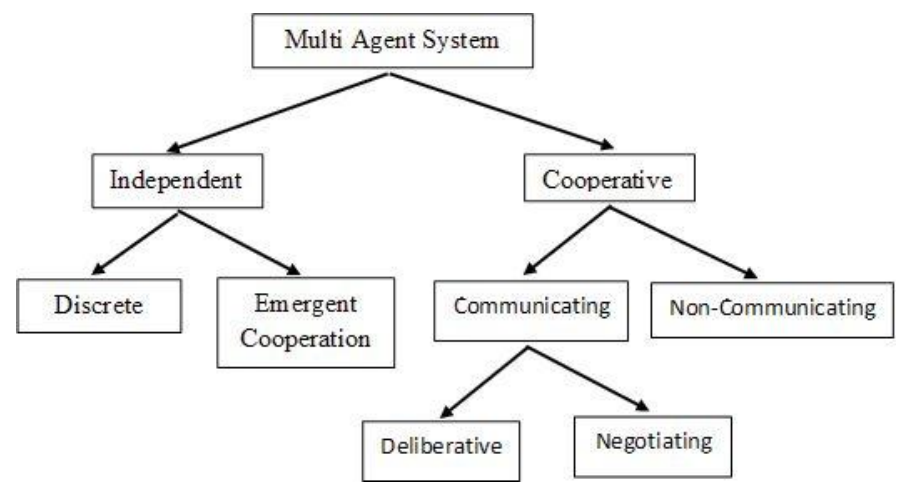

Fig. 2. Multi Agent Hierarchy.

\section{MULTI AGENT CONTROL STRATEGY FOR DC NANO-GRID}

The DC Nano-Grid should be able to function in the following modes, depending on the load demand, the battery charging status, the energy generated from the PV and the condition of the power grid.

- Mode 1 Solar power + Grid power = Load Demand

- Mode 2 Solar Power = Load Demand + Battery Power

- Mode 3 Solar Power = Load Demand + Grid power

- Mode 4 Solar Power + Battery Power = Load Demand

- Mode 5 Battery Power = Load Demand

- Mode 6 Grid Power = Load Demand

To switch from one mode to another mode depending upon the conditions will be decided by particular agents given below,

- Source Agent

- Battery Agent

- Grid Agent

- Load Agent

\section{A. Source Agent}

Source agent is the maximum power point tracking (MPPT) controller for highest PV energy with variable atmospheric circumstances. Incremental conductance algorithm is used for maximum PV panel power point monitoring. In the incremental conductance method, the controller measures incremental changes in the current and voltage of the PV array to predict the impact of a voltage change. This technique needs more computation in the controller, but can monitor evolving circumstances faster than perturbing and observing technique $(\mathrm{P} \& \mathrm{O})$. Like the $\mathrm{P} \& \mathrm{O}$ algorithm, it can generate energy output oscillations. 


\section{B. Battery Agent}

Battery agent is used to connect or disconnect the battery to the dc link depending upon state of charge of battery, charging or discharging condition of the battery and received command from the grid agent. After that, based on the battery SoC condition the control signal send ON/OFF command to the grid. The battery agent algorithm shown in algorithm 1 .

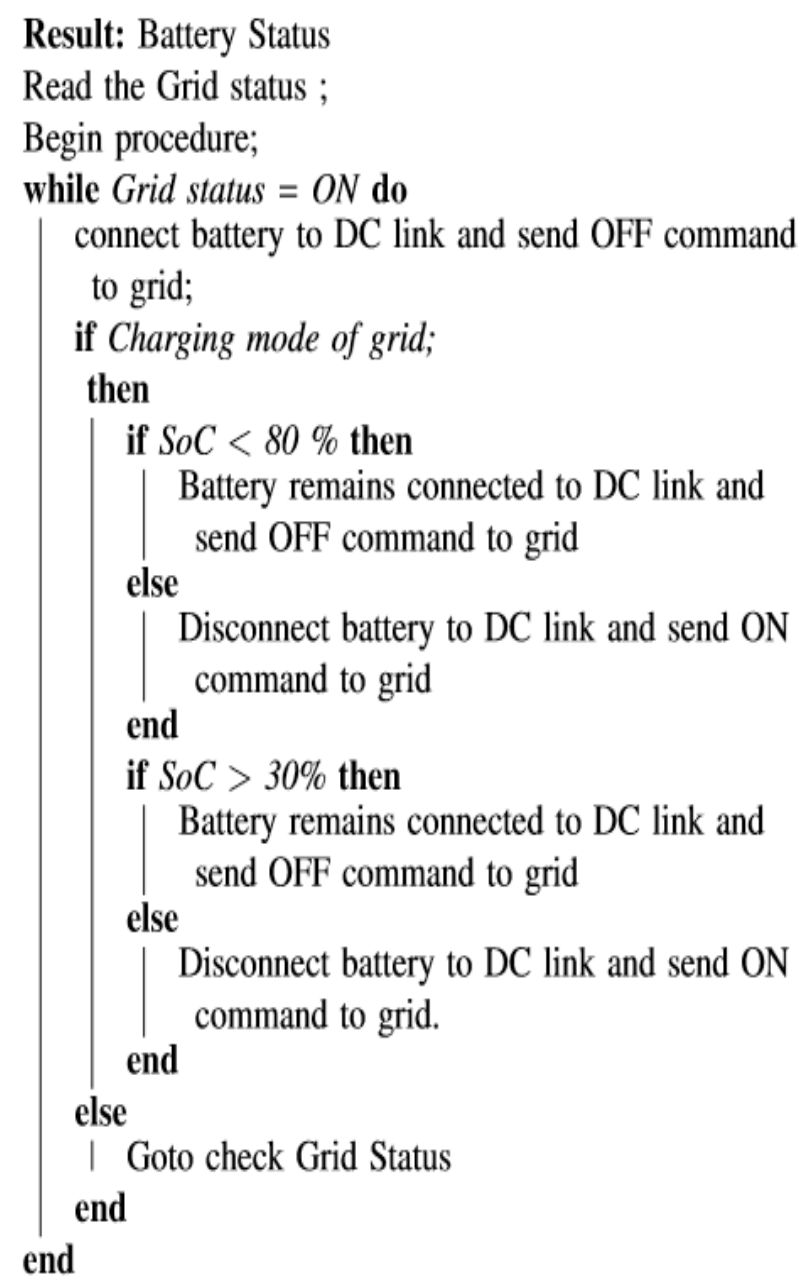

end Procedure;

Algorithm 1: Battery Agent algorithm

\section{Grid Agent}

Grid agent is used to connect or disconnect the Grid to the dc link depending upon state of charge of battery, rectifier or inverter mode of the bidirectional converter and received command from the battery agent. After that, based on the battery SoC condition the control signal send connect/disconnect command to the battery. The grid agent algorithm shown in algorithm 2 .

\section{Load Agent}

Load agents are used to control loads connected to dc links to utilize battery effectively and to minimize power taken from the AC grid. Load agents disconnects the respective loads having least priority when either battery is discharging or power is being taken from AC grid. The load agent algorithm shown in algorithm 1 . Here, the load agent controls the household appliances ON and OFF mode.

\section{Result: Grid Status}

Read the Battery status ;

Begin procedure;

while Battery status $=O N$ do

Turn ON grid to DC link and send disconnect command to battery agent;

if Rectification mode of battery; then

if $\mathrm{SoC}<30 \%$ then

Grid remains $\mathrm{ON}$ and send disconnect command to battery Agent

else

Turn OFF grid and send connect command to battery agent

end

if $\mathrm{SoC}>80 \%$ then

Grid remains $\mathrm{ON}$ and send disconnect

else command to battery agent

Turn off grid and send connect command to battery agent end

else

Goto check battery Status

end

end

end Procedure;

Algorithm 2: Grid Agent algorithm

Result: Automatic load Status

Read the manual load status ;

Begin procedure;

while Load status $=O N$ do

if Non-Rectification mode of grid;

then

if Non-battery discharge then

I Load remains connected

end

if Priority $=0$ then

| Disconnect load

end

else

I Load remains connected

end

load remains connected;

end

end Procedure;

Algorithm 3: Load Agent algorithm

\section{SIMULATION RESULTS}

DC nano grid configuration given in figure is simulated in MATLAB Simulink. Multi agents are modelled by MATLAB coding using MATLAB function block. Back to back connected IGBT module is used as bidirectional switch to connect or disconnect battery and three phase grids to the dc link controlled by respective battery agent and grid agent.

\section{A. Loads}

DC loads are modelled as equivalent resistive loads. Voltage rating, quantity and power rating of loads is given in table. I. 
Table- II: Load Details

\begin{tabular}{|c|c|c|}
\hline Name of the Load & Qty & Power in (W) \\
\hline Washing machine & 1 & 512 \\
\hline Air conditioner & 1 & 700 \\
\hline Water pump & 1 & 350 \\
\hline Hair Drier & 1 & 425 \\
\hline Refrigerator & 1 & 80 \\
\hline Mixer & 1 & 200 \\
\hline Dough maker & 1 & 150 \\
\hline Grinder & 1 & 120 \\
\hline Water Heater & 1 & 600 \\
\hline Oven & 1 & 300 \\
\hline Coffee maker & 1 & 135 \\
\hline Vacuum cleaner & 1 & 95 \\
\hline Ceiling fan & 3 & 22 \\
\hline Television & 1 & 30 \\
\hline Computer & 1 & 170 \\
\hline LED & 3 & 7 \\
\hline CFL & 3 & 12 \\
\hline Tube light & 3 & 9 \\
\hline
\end{tabular}

\section{B. Simulation Mode}

For a full day 24-hour operation, 24 hours are compressed in to 48 seconds i.e. 2 seconds are equal to one hour. Irradiation is variable from 0 to 24 seconds and for rest 24 seconds irradiation is zero i.e. solar power is not available. Temperature is constant at $25 \operatorname{deg} C$ throughout the simulation time. The MATLAB Simulink model is shown in Fig.3

\section{Mode of Operation}

DC nano grid is having six modes of operation which will be controlled by the multi agent. DC link feeds dc loads either in three phase grid connected mode or battery connected mode to maintain constant dc link voltage at 24 volts. 12 -volt dc loads are supplied by 24/12-volt buck converter connected to DC link. waveforms of different powers such as solar power in 4, battery power in Fig. 6, active power from grid in Fig. 7, reactive power from grid in Fig. 8 and load demand for a single house is shown in Fig.9 are given below,

- Mode 1: Solar power + Grid power = Load Demand: Initially battery is discharged i.e. State of charge of battery is shown in Fig. 10, which is less than $30 \%$. Battery is not connected to the DC link and three phase grids is in ON state. Grid agent is continuously sending "disconnect" command to the battery agent. Available solar power is not enough to meet the load demand, so bidirectional converter is operating in rectifier mode. At time T1 in Fig. 7, solar power increases more than load demand so bidirectional converter changes from rectifier to inverter mode. Grid agent detects the reversal of power and checks SOC of battery. As SOC is less than $30 \%$ so grid agent turns OFF the grid and sends "connect" command to the battery agent.

- Mode 2: Solar Power = Load Demand + Battery Power: Mode 2 starts at the end of mode 1 at time T1 in Fig.7. Battery agent receives "connect" command from grid agent and connects battery to the DC link and sends "OFF" command to the Grid agent. Now available solar power is feeding loads as well as charging the battery. At time T2 battery SOC becomes greater than $80 \%$, so battery agent will disconnect the battery and send "ON" command to the grid agent.

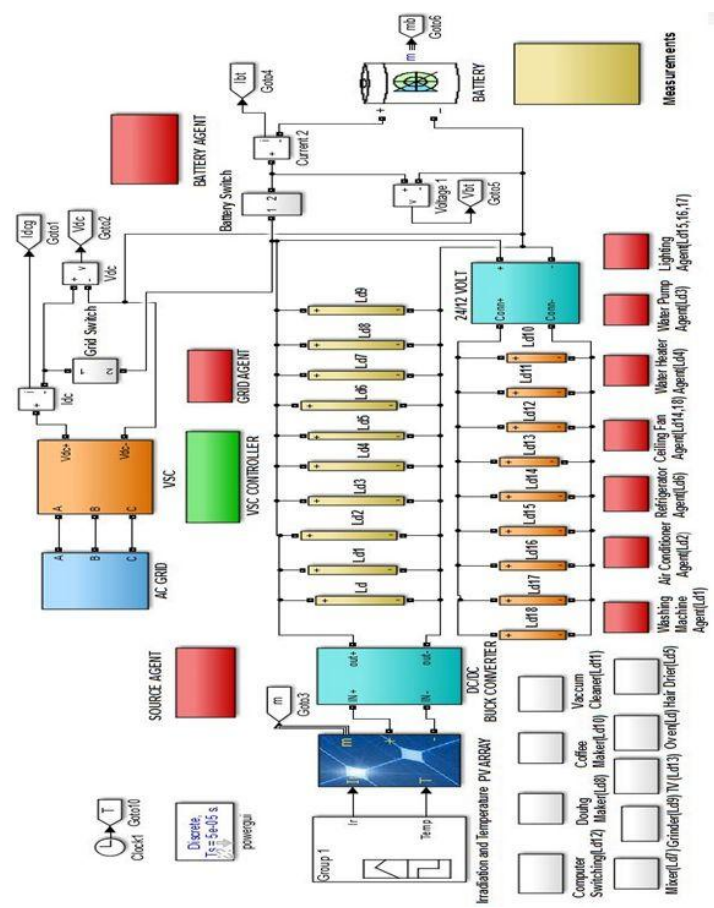

Fig. 3. MATLAB Simulation Model of the Proposed System.

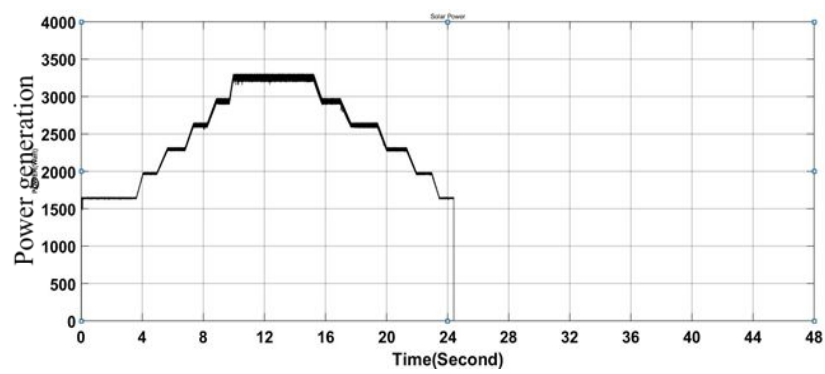

Fig. 4. Solar Power Generation.

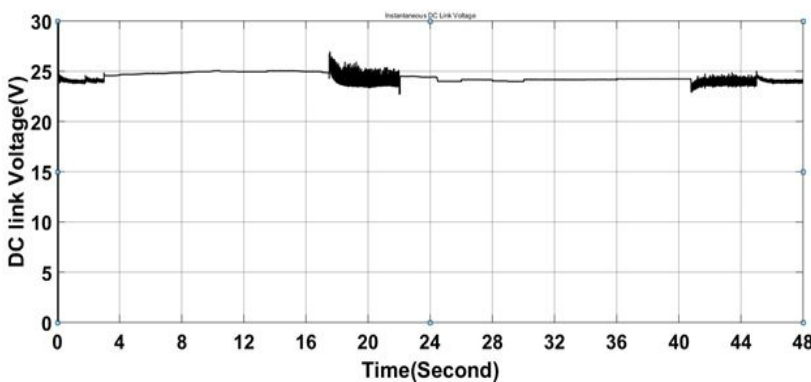

Fig. 5. Instantaneous DC link voltage.

- Mode 3: Solar Power = Load Demand + Grid power: Mode 3 starts at time T2 in Fig.7. Grid agent receives "ON" command from battery agent and turns ON grid to the DC link. Solar power is more than load demand, so bidirectional converter operates in inverter mode. In this mode, solar power is feeding load and exporting power to the three- $\mathrm{ph}$ a s e ac gird. At time T3 solar power becomes less than load demand so bidirectional converter changes its operation from inverter to rectifier mode. Grid agent detects the operating mode and checks SOC of battery. As battery SOC is greater than $30 \%$, it can deliver power to the loads so no need to import power from ac grid. Grid agent sends "connect" command to the battery agent and turns off grid to the DC link. 


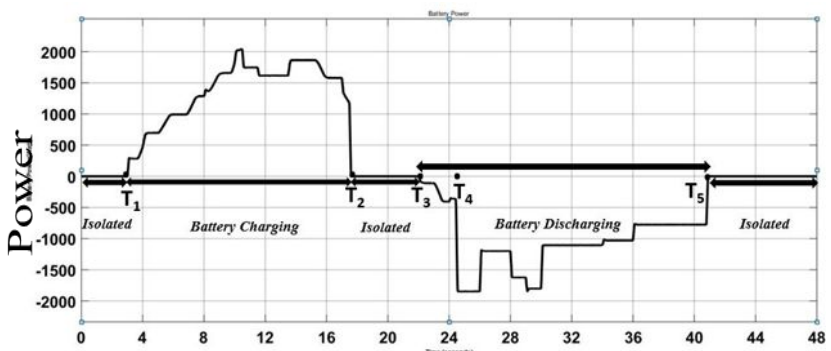

Fig. 6. Battery Power.

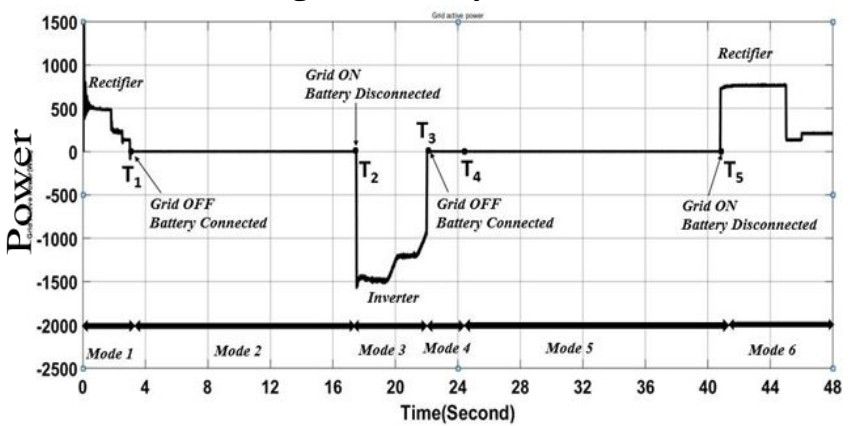

Fig. 7. Active Power from Grid.

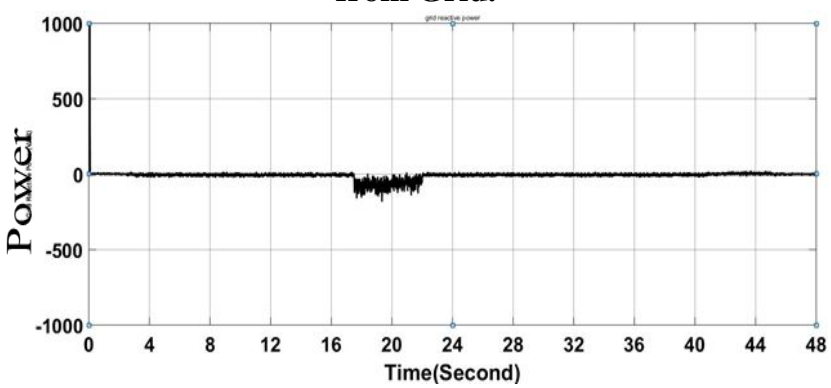

Fig. 8. Reactive Power.

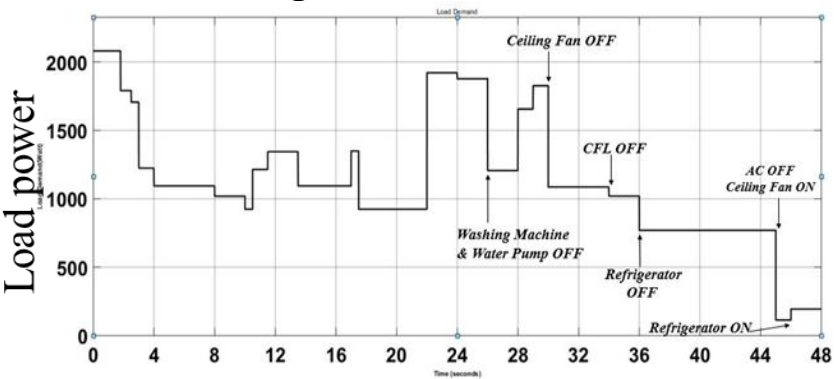

Fig. 9. Load demand.

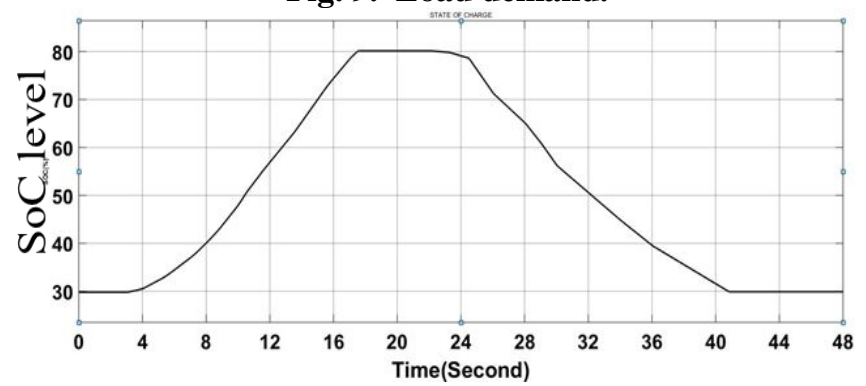

Fig. 10. State of Charge of Battery.

- Mode 4: Solar Power + Battery Power = Load Demand: Mode 4 starts at time T3 in Fig.7. Battery agent receives "connect" command from grid agent and connects battery to the DC link and sends "OFF" command to the Grid agent. Now load demand is met by both available solar power and battery. In this mode battery is discharging. At time T4, available solar power becomes zero and operation enters in to mode 5 .
- Mode 5: Battery Power = Load Demand:

Mode 5 starts at time T4 in Fig.7. In this mode battery is discharging to feed loads. In this mode load demand is met only by battery. At time T5 SOC of battery becomes less than $30 \%$, battery agent will disconnect battery and send "ON" command to the grid agent.

- Mode 6: Grid Power = Load Demand: Mode 6 starts at time $\mathrm{T} 5$ in Fig.7. Grid agent receives "ON" command from the Battery agent and turns ON ac grid to the dc link and sends disconnect command to the battery agent. In this mode load demand is met by only three phase ac grid. When solar power becomes available operation enters from mode 6 to mode 1 .

In Fig. 4 solar power is shown up to $24.5 \mathrm{sec}$ there is variable solar power and from 24.5 to 48 second solar power is zero. DC link voltage is to be maintained constant at 24 -volt, $5 \%$ variation is acceptable, in Fig. 5 instantaneous DC link voltage is shown, which is within permissible range through all modes of operation. DC nano grid is neither exporting nor importing any significant amount of reactive power from the ac grid as shown in Fig. 8, operating status of bidirectional converter rectifier or inverter is not affecting utility grid with respect to reactive power. DC nano grid is only exporting or importing active power to the utility grid.

\section{APPLICATION IN RURAL AREAS}

More than a billion individuals around the world still do not have access to fundamental contemporary energy services such as electric lighting in their homes. Most of these individuals live in distant rural regions, making expansion of domestic electrical grids prohibitively costly to satisfy their requirements. DC nano grid can be operated in both standalone mode for remote area and utility grid connected mode where utility grid is available. DC nano grid is a simple and cost- effective way to meet a portion of your own electricity demand separately, decrease the electric bill, create savings, add to the exploitation of renewable energy sources and contribute to the conservation of the environment by decreasing greenhouse gas emissions. Such an opportunity is obviously reserved to those users which have plenteous space for installing a PV system, in rural areas citizens have sufficient space to install PV plants. Citizens in rural areas who have a large area oversize their photo-voltaic plants to generate electricity for those living in downtown and who cannot install a photo-voltaic plant on the rooftop. Citizens can generate energy and engage in the electricity market, selling energy to formers. The electricity market is available to all people who become aggregate true market operators. Such a transition would have an instant financial return, relying on price margins between wholesale and retail rates.

\section{CONCLUSION AND FUTURE SCOPE}

In this project, the development of a smart DC nano grid was intended and created using multi-agent system to provide a household with a high level of convenience, power effective- ness and energy cost savings. 
An overall idea of the DC Nano- grid is created using a multi-agent system that allows agents to intercommunicate, interact and hash out with energy resources and devices in the DC Nano-grid that attain maximum overall system energy efficiency and minimum electricity bills and smooth control of different operating modes. DC nano grid is operating either in battery connected mode or utility grid connected mode, controlled by battery agent and grid agent to maintain constant dc link voltage. Energy imported from utility grid is minimized by load shedding during rectifier mode of bidirectional converter, in addition load shedding is also done when battery is discharging to increase discharge time of battery. Load shedding is done on the basis of fixed priority of loads. Priority of loads is fixed with consideration of minimum consumer trouble.

\section{FUTURE SCOPE:}

In this project fixed priority is given to the loads, automatic scheduling of the loads with various priority algorithms can be implemented. For hardware implementation of agents Arduino board will be a cost effective and reliable solution. For communication among the agents wi-fi will be suitable communication medium as a home area network.

\section{REFERENCES}

1. M. Haidar, K. Muttaqi, and D. Sutanto," Smart grid and its future perspectives in Australia," Renewable and Sustainable Energy Reviews, vol. 51, 2015, pp. 1375-1389.

2. S. Uski and I. Kim, "Assessment of wind power impact on power system transmission losses," in IEEE PES Innovative Smart Grid Technologies, Europe,2014, pp. 1-5.

3. P. Friedlingstein, R. M. Andrew, J. Rogelj, et. Al., "Persistent growth of $\mathrm{CO} 2$ emissions and implications for reaching climate targets", Nature Geoscience, Vol 7, 2014, pp. 709-715.

4. Akinyele, R. Rayudu, and N. Nair, "Global progress in photovoltaic technologies and the scenario of development of solar panel plant and module performance estimation application in nigeria," Renewable and Sustainable Energy Reviews, vol. 48, 2015, pp. 112-139.

5. H. Holtorf, T. Urmee, M. Calais, and T. Pryor, "A model to evaluate the success of solar home systems," Renewable and Sustainable Energy Reviews, vol. 50, 2015, pp. 245-255.

6. Burmester, D., Rayudu, R., Seah, W., and Akinyele, D, "A review of nanogrids topologies and technologies," Renewable and Sustainable Energy Reviews, Vol 67, 2017, pp. 760-775.

7. M. Biabani, M. A. Golkar, A. Johar, and M. Johar, "Propose a home demand-side-management algorithm for smart nano-grid," in Proc. 4th Power Electron. Drive Syst. Tech. Conf. (PEDSTC), Tehran, Iran, pp.

8. 487-494, 2013.

9. Toshihisa Funabashi (2016), "Integration of Distributed Energy Resources in Power Systems, Implementation, Operation and Control," 1st Edition, Academic press.

10. Cecati, C., Khalid, H. A., Tinari, M., Adinolfi, G., and Graditi, G., "DC Nanogrid for renewable sources with modular DC/DC LLC converter building block. IET Power Electronics, Vol 10(5), 2017 pp. 536-544.

11. Miguel A. Sanz-Bobi Use. (2014), "Operation and Maintenance of Renewable Energy Systems: Experiences and Future approaches," springer.

Kantamneni, A., Brown, L. E., Parker, G., and Weaver, W. W., "Survey of multi-agent systems for microgrid control," Engineering Applications of Artificial Intelligence, Elsevier, Vol. 45, 2015, pp. 192-203.

12. 12.Khan, Muhammad Waseem and Wang, Jie, "The research on multi- agent

system for microgrid control and optimization," Renewable and Sustainable Energy Reviews, Elsevier, vol. 80(C), 2017, pp. 1399-1411

13. Xie, J., and Liu, C.-C., "Multi-agent systems and their applications," Journal of International Council on Electrical Engineering, Taylor and Francis Vol. 7(1), 2017, pp. 188-197.

14. Anvari-Moghaddam, Amjad; Rahimi-Kian, Ashkan; Mirian, Maryam
S.; Guerrero, Josep M., "A Multi-Agent Based Energy Management Solution for Integrated Buildings and Microgrid System," Applied Energy, Elsevier, Vol. 203, 2017, pp. 41-561.

15. L. Wang, Z. Wang, and R. Yang, "Intelligent multiagent control system for

energy and comfort management in smart and sustainable buildings," IEEE Transactions on Smart Grid, vol. 3(2), 2012, pp. 605-617.

\section{AUTHORS PROFILE}

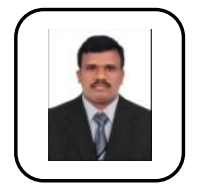

Periyasamy Muthuvel was born in Cuddalore, Tamil Nadu, India, on May 3, 1987. He received the B.E. degree from the Madras Institute of Technology Campus (Anna University), Chennai, India, in 2009, the M.E. degree in Power Electronics and Drives from the Government College of Technology (Anna University), Coimbatore, India, in 2012, and the Ph.D. degree from the National Institute of Technology, Tiruchirappalli, India, in 2018. He is currently an Assistant Professor at the Department of Electronics and Instrumentation Engineering, Kalasalingam Academy of Research and Education, Virudhunagar, where he has been since March 2018. His research interests include power-electronic applications in renewable-energy systems, Battery \& Energy Management System, Electric vehicle and Smart DC appliances.

Dr. P. Muthuvel, is a Member of IEEE and an Associate Member of Institution of Engineers, India. 